\title{
Simulation and Experimental Verification for a 52 GHz Wideband Trapped Surface Wave Propagation System
}

\author{
Jixiang Wan, Kin Fai Tong, Senior Member, IEEE, Chi Hou Chan, Fellow, IEEE
}

\begin{abstract}
Trapped Surface wave (TSW) provides a flexible two-dimensional wireless solution when compared to existing wired and free space communications systems. This work aims to provide the theoretical guidance, supported by simulation techniques and experimental verifications, for i) designing the wideband and highly efficient rectangular aperture TSW transducers, and ii) selecting the best reactive surface impedance for high efficiency. Firstly, a method for computing the TSW excitation efficiency is proposed for the first time and it can be applied to different kinds of reactive surface geometries and transducer apertures. Then the relation between the TSW excitation efficiency, surface reactance and aperture height is derived. Then the maximum excitation efficiency, the corresponding optimal surface reactance and aperture height are presented. Further, the relation between the TSW angular coverage and aperture width is determined. These studies show that the aperture height determines mainly the TSW excitation efficiencies while the aperture width controls the TSW angular coverage, hence the two aperture parameters of a transducer can be determined independently. Finally, an experiment setup, based on the provided guidelines, has been built to demonstrate a $52 \mathrm{GHz}$ wide 3dB-transmission-bandwidth for high performance communications systems.
\end{abstract}

Index Terms - Surface wave, ultrawideband antenna, aperture

\section{INTRODUCTION}

$\mathbf{T}$ he Trapped Surface Wave (TSW) is the one that propagates along a dielectric coated planar conductor without radiation [1]. TSW is very similar to Zenneck wave [2] as both fields of the waves decay exponentially ( $\propto$ $r^{-1}$, where $r$ is range) in the propagating direction, but Zenneck wave is due to a Brewster zero, rather than a surface wave pole [1], [3]. Many theories and experiments about TSW, including several different kinds of reactive surfaces and transducers [4] [13], especially on how to excite TSW efficiently were reported between 1950s - 1970s. However, the TSW seems to be forgotten between the 1980s and 2000s. Recently, traditional wired communications systems and space wave wireless communications systems are facing bottlenecks in some applications such as ultra-wideband communications over the sea [14], wireless body area networks (WBANs) [15], millimeter-wave wireless interconnects for networks-on-chip [16]. Therefore, the TSW technology has attracted researchers' attention again because of its unique characteristics:

1) When compared to wired communications systems, it can provide one-to-many or many-to-one communication and higher reliability due to its characteristic of propagating along a robust two-dimensional surface [17].

2) When compared to wireless communications systems based on traditional space wave, it can provide three key advantages:

i) Enhanced power savings as its power is mainly spread over a two-dimensional surface instead of a threedimensional sphere;

ii) Non-line-of-sight communication because of its guided wave characteristic that propagates along a surface; and

iii) Excellent EMC requirements because of its unique nonradiating characteristic.

Fig. 1 suggested a possible TSW wireless communications system. It comprises of a reactive TSW surface and a number of communication devices. The devices are communicating with each other by means of TSW surface through their TSW transducers. To utilize this system, the following two questions must be answered:

1) Can't the guided wave be excited efficiently into TSW by a suitable transducer and reactive surface?

2) How much the TSW transducers angularly covers over the reactive surface?

The existing study concentrates mainly on the first technology. The TSW excitation efficiency depends on both the transducer and reactive surface. Different surfaces have been studied including dielectric-coated metal surface [7], corrugated metal surface [13] and periodic structures surface (periodic structured surface or periodic structure) [18]. As for transducers, a horn [4], dipole [5], [6] or an end-fire array of slots [8] has been presented. The measurements of TSW excitation efficiency with these surfaces and transducers have been performed. However, these works have only involved single frequency point or narrow band applications. Aperture antennas, such as rectangular horn antennas, are good candidates for high efficiency and very wideband TSW communications. To our best knowledge, the detailed design method of rectangular aperture TSW transducer has not been reported. As for the second technology, the general theory has not been built completely so far. The aim of this paper is to work out these issues by studying the excitation efficiency of a

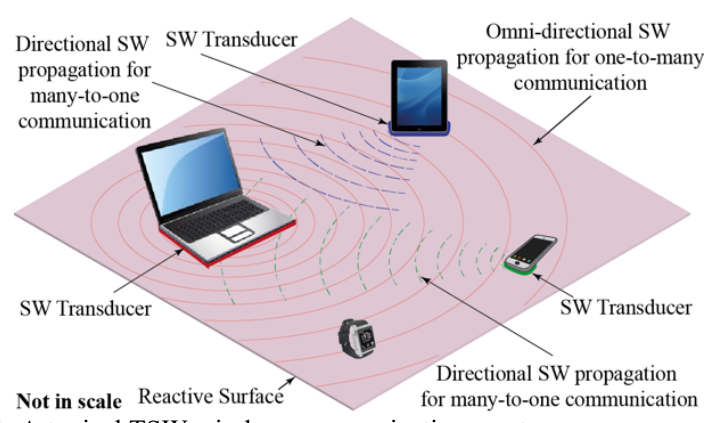

Fig. 1. A typical TSW wireless communications system. 
rectangular aperture transducer on lossless reactive surface. The major contributions of this paper are as follows:

1) A simple TSW decision condition is derived using a logarithmic expression. The TSW power with different vertical height is quantitated.

2) A valid and general method of computing TSW excitation efficiency is presented and applicable for all kinds of reactive surface and transducer.

3) The relation between the TSW excitation efficiency and surface reactance and aperture height is set up.

4) The relation between TSW angular coverage and aperture width is determined.

\section{ThE TSW EXCITATION EFFICIENCY AND APERTURE HEIGHT}

\subsection{The definition of TSW Excitation Efficiency}

The TSW excitation efficiency is crucial to a TSW communications system. In order to excite pure TSW without radiation, Barlow and Brown stated that in addition to a lossless surface, an ideal transducer of infinite height with exponential decay $E$-field distribution along the height is required [12]. Obviously, it is not feasible to have an infinite height transducer. Even in the case of finite height, it is almost impossible to build up an exponential decay $E$-field along the height. This is because most rectangular aperture transducers are usually excited by the fundamental $\mathrm{TE}_{10}$ mode which has uniform $E$-field distribution along the height. Therefore, it is inevitable that for a non-ideal TSW transducer, some space waves and reflected waves at the aperture-air boundary will be present because of the mismatch between the practical uniform aperture field distribution and non-ideal exponential decayed TSW field distribution as shown in Fig. 2. We therefore define the TSW excitation efficiency $\eta_{s}$, as

$$
\eta_{s}=\frac{P_{s u r}}{P_{t o t}}=\frac{P_{s u r}}{P_{s p a}+P_{s u r}+P_{r e f}}
$$

where $P_{\text {tot }}$ represents the total incident power, $P_{\text {spa }}$ represents the space wave power, $P_{\text {sur }}$ represents the TSW power, and $P_{\text {ref }}$ represents the reflected wave power.

In [9], the reflected wave power $P_{\text {ref }}$ is neglected. This is because the analytical model of the transducer is adopted in their work. However, a 3D-model is used in this paper and the effect of the reflected wave power $P_{r e f}$ will be considered. More accurate solutions will be obtained.

\subsection{The computing method of TSW excitation efficiencies}

To obtain the TSW excitation efficiency, the TSW power $\left(P_{\text {sur }}\right)$ must be determined first. To the authors' best knowledge,

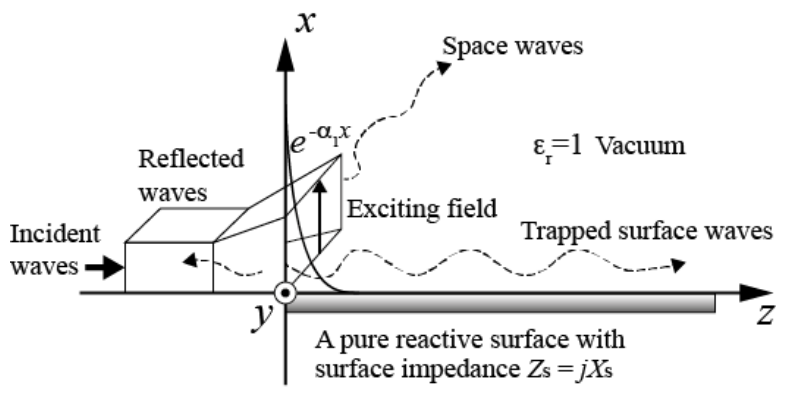

Fig. 2. Excitation of TSW on a pure reactive surface. there are no commercial electromagnetic tools which can directly calculate TSW power. With the help of the TSW theory, an efficient computing method is represented in this paper.

\section{A. The decayed characteristic of TSW vertically away from the reactive surface}

As shown in Fig. 2, the reactive surface lies on the $y z$-plane at $x=0$ and the region $x>0$ is air. Assume a TSW is bound to the reactive surface and propagates in the $z$-direction with propagation constant $k$. For a TM polarized TSW, the $E$-field is decayed in the $+x$-direction and has the following form in the upper half space [9]:

$$
E_{x}=E_{x 0} e^{-\alpha_{1} x}
$$

where the factor $e^{j \omega t-j k z}$ has been omitted for simplicity, $E_{x 0}$ is the amplitude of the $E$-field and $\alpha_{1}$ is decay constant in the $+x$-direction. Here we assume $E_{x 0}=1$ without loss of generality.

For a pure reactive, i.e. a lossless surface $\left(Z_{s}=j X_{s}\right)$, then

$$
\alpha_{1}=\omega \varepsilon_{0} X_{s}
$$

Where $\omega$ is the angular frequency, $\varepsilon_{0}$ is the permittivity in vacuum, and $X_{s}$ is the surface reactance.

From (2), the $E$-field expressed in $\mathrm{dB}$, will be

$$
20 \log \left(E_{x}\right)=-8.7 \alpha_{1} x(d B)
$$

Let $h_{e}$ represent the height when the E-field decaying to $1 / e$ of $E_{x 0}$, from (2) we can get

$$
h_{e}=\frac{1}{\alpha_{1}}
$$

Substituting (5) into (4) yields:

$$
20 \log \left(E_{x}\right)=-8.7 \frac{x}{h_{e}}(d B)
$$

From (6), it shows that the $E$-field decay ratio with normalized vertical height is $-8.7 \mathrm{~dB}$ per $h_{e}$ which is also plotted in Fig. 3.

Further, using (2), the percentage of TSW power at different heights can be obtained:

$$
P_{i}=\int_{(i-1) h_{e}}^{i \times h_{e}}\left(E_{x 0} e^{-\alpha_{1} x}\right)^{2} d x \quad i=1 \sim 4
$$

Here, $P_{1}, P_{2}, P_{3}$, and $P_{4}$ represent the percentage of TSW power in the range of $0 \leq x \leq h_{e}, h_{e} \leq x \leq 2 h_{e}, 2 h_{e} \leq x \leq 3 h_{e}$ and $3 h_{e} \leq x \leq 4 h_{e}$ respectively and is shown in Fig. 4 . It can be seen that when $x \geq 2 h_{e}$, the TSW power in the range of $[0, x]$ will more than $98.2 \%$ of the total TSW power.

\section{B. The computation of TSW power}

The TSW power $\left(P_{\text {sur }}\right)$ can be evaluated by:

$$
P_{\text {sur }}=\frac{1}{2} \int_{S} \operatorname{Re}\left(E_{\text {sur }} \times H_{\text {sur }}^{*}\right) d s
$$

Where $\operatorname{Re}[]$ is the real part of a complex quantity, $S$ is the TSW power integration boundary, $E_{\text {sur }}$ is the TSW electric field, $H_{\text {sur }}{ }^{*}$ is the complex conjugate of TSW magnetic field. 


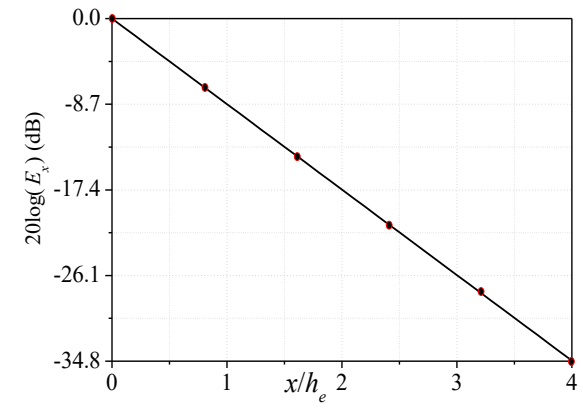

Fig. 3. TSW $E$-field (dB) decays linearly in x-direction.

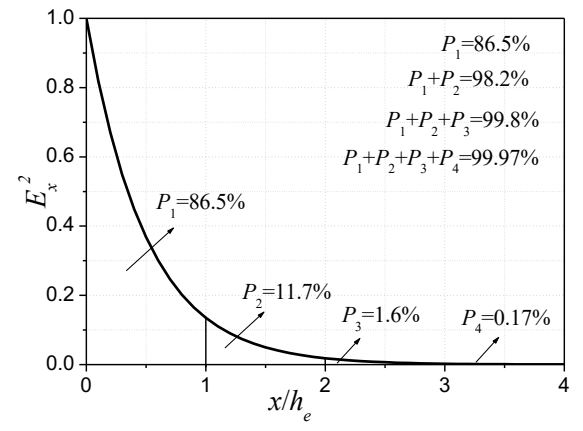

Fig. 4. TSW Power Percent with vertical height.

Given the incident total power $P_{t o t}$, the values of $E_{\text {sur }}$ and $H_{\text {sur }}{ }^{*}$ can be retrieved from the electromagnetic simulation. To determine $P_{\text {sur }}$, we only need to define a suitable TSW power integration boundary $S$. Since a TSW propagates along a reactive surface while a space wave power decays with the square of distance from the source, therefore we set up the TSW power integration boundary from the source and the edge where the space wave is negligible.

To ensure the space wave power is negligible, the TSW power integration boundary must meet the following two conditions:

Condition 1: The E-field variation of integration boundary satisfies $-8.7 \mathrm{~dB} / h_{e}$ to guarantee a pure TSW and avoid the disturbance from the space wave.

Condition 2: The height of integration boundary, $h_{s}$, must be greater or equal to $2 h_{e}$. This will guarantee that the TSW power is more than or equal to $98.2 \%$ of the total TSW power, only insignificant amount of TSW is not considered.

To verify these two conditions, we can set some $E$-field sampling lines marked " $E$-field Line 1" and " $E$-field Line 2 " in the simulation model as shown in Fig. 5 to investigate the $E$ field decay variation along these vertical lines. Ansys HFSS was used in the EM simulation models. As an example, a typical $E$-field distribution along the sampling line of the TSW integration face is shown in Fig. 8. It can be seen that the $E$ field distribution is satisfied well with the TSW decayed rule of $-8.7 \mathrm{~dB} / h_{e}$ when $h_{s} \leq 4 h_{e}$. Hence the integration face is reasonable and we can choose $2 h_{e} \leq h_{s} \leq 4 h_{e}$ as the height of

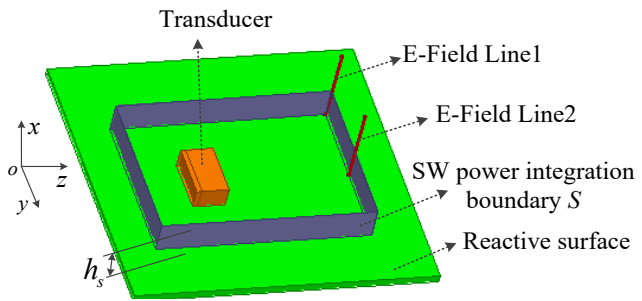

Fig. 5. The simulation model of computing TSW excitation efficiency. integration face in this example. After determining the integration face, the TSW power $\left(P_{\text {sur }}\right)$ can be obtained by using (8).

\section{The computation of the radiated and reflected power}

The reflected power $P_{\text {ref }}$ caused by the mis-match at the aperture-air boundary can be expressed as

$$
P_{r e f}=P_{t o t}|\Gamma|^{2}
$$

where $\Gamma$ represents the reflection coefficient at the feed port of the transducer. Therefore, the power coming out from the transducer $P_{s u r}+P_{s p a}$

$$
P_{s u r}+P_{s p a}=P_{t o t}\left(1-|\Gamma|^{2}\right)
$$

\section{The computation of the TSW excitation efficiency}

After determining the TSW power $P_{\text {sur }}$ and the reflected wave power $P_{\text {ref }}$, the TSW excitation efficiency can be obtained by using (1). If neglecting the reflected wave power as in [9], the TSW excitation efficiency will be given as

$$
\eta_{s}=\frac{P_{s u r}}{P_{s u r}+P_{s p a}}
$$

\subsection{The relation between TSW excitation efficiency and aperture height with pseudo $3 D$ Model for a millimeter-wave system}

The rectangular aperture of a transducer basically has two design parameters: the height $\left(H_{a}\right)$ and width $\left(W_{a}\right)$. As discussed in the later section, the height is related to the TSW excitation efficiency and the width mainly decides the TSW angular coverage in the $y z$-plane. Therefore, a pseudo 3D simulation model, with width equals to one free space wavelength $\left(\lambda_{0}\right)$, is set up to calculate the relation among the TSW excitation efficiency, surface reactance and the aperture height, as shown in Fig. 6. Perfect magnetic boundaries in the two $x z$-planes [14] are considered to save simulation time. The reactive surface is defined by impedance boundary in HFSS [19]. Wave ports are used to produce the uniform $E$-field distribution by a small vertical aperture with initial height $h_{e}(5)$. For a given surface reactance $X_{s}$, the simulations are performed at $60 \mathrm{GHz}$ frequency band.

\section{A. The verification of TSW power integration face}

To verify the TSW power integration face, four TSW power integration faces with different heights, $h_{e}, 2 h_{e}, 3 h_{e}$ and $4 h_{e}$, are set up in Fig. 6. A red $E$-field line is inserted to check if the $E$-field along the line satisfies the two TSW conditions mentioned in section IIB.

As an example, a reactive surface with $X_{s}=j 200 \Omega$ is preliminary selected at $60 \mathrm{GHz}$ and $h_{e}$ is found to be $1.5 \mathrm{~mm}$ based on (5). Fig. 7 shows the $E$-field distribution along TSW propagation direction in the $x z$-plane. Fig. 8 presents the $E$-field distribution along the central red line. It can be observed that the $E$-field is satisfied well with $-8.7 \mathrm{~dB} / h_{e}$ when $h_{s} \leq 4 h_{e}$. And the TSW power propagates through integration face of the first three heights are computed and summarized in Table I. As shown in Fig. 4, the discrepancy between the theoretical total TSW power and that with the height of $4 h_{e}$ is only $0.03 \%$, which is negligible, so the power percentages are evaluated by using (10). Good agreements between theoretical and simulation results are observed. This indicates that the integration face is 


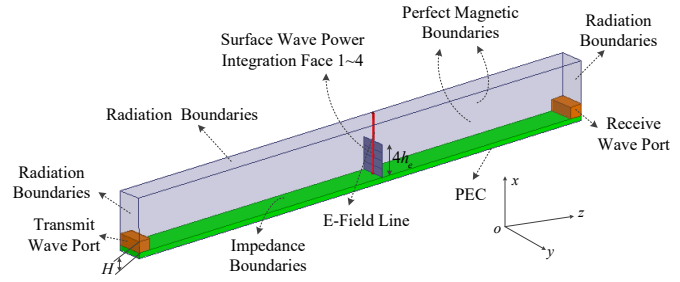

Fig. 6. The pseudo 3D simulations setup.

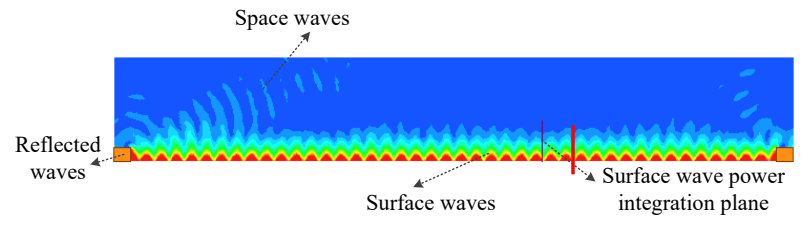

Fig. 7. $E$-field distribution in $y z$-plane with $X_{s}=j 200 \Omega$ at $60 \mathrm{GHz}$ in the pseudo 3D-model (the color scale is shown in Fig. 15).

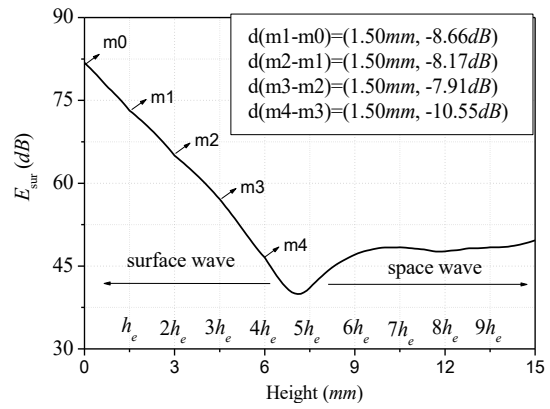

Fig. 8. Normalized $E$-field distribution along the central line $(y=0)$ in the $x z$-plane with $X_{s}=j 200 \Omega$ at $60 \mathrm{GHz}$.

valid and we can choose $2 h_{e} \leq h_{s} \leq 4 h_{e}$ as the height of integration face in this example.

$$
P_{\mathrm{i}} \% \approx P_{\mathrm{i}} /\left(P_{1}+P_{2}+P_{3}+P_{4}\right) \times 100 \%(i=1,2 \text {, and } 3)(12)
$$

TABLE I TSW POWER WITH DIFFERENT INTEGRATION HEIGHT

\begin{tabular}{|c|c|c|c|}
\hline No. & Height & $\begin{array}{c}\text { Simulation } \\
\text { results }\end{array}$ & Theoretical results from (12) \\
\hline 1 & $h_{e}$ & $87.0 \%$ & $86.5 \%$ \\
\hline 2 & $2 h_{e}$ & $98.4 \%$ & $98.2 \%$ \\
\hline 3 & $3 h_{e}$ & $99.8 \%$ & $99.8 \%$ \\
\hline
\end{tabular}

\section{B. Simulated results and discussion}

After defining the TSW efficiency and validating the height for the integration area, we further investigate the relation between different transducer aperture heights $\left(H_{a}\right)$ and surface reactance $X_{s}$ in the millimeter wave frequency range. The corresponding TSW excitation efficiency is computed by (1) and (11) respectively. Fig. 9 shows the maximum TSW excitation efficiency as a function of surface reactance $X_{s}$. It can be seen that

1) As shown in Fig. 9, the TSW excitation efficiency will increase with surface reactance $X_{s}$. If the reflected wave power $\left(P_{r e f}\right)$ is neglected, the TSW efficiency will be above $95 \%$ when surface reactance $X_{s}$ is higher the $j 200 \Omega$. This is in good agreement with the magnetic line source results reported in [11].

2) When the aperture field distribution mismatches with expected TSW field distribution, reflected wave will be incurred, in addition to space wave. The $\mathrm{S}_{11}$ at the transducer aperture is included in Fig. 9 for reference as well. The existence of reflected wave results in decrease of TSW excitation efficiency. It is more serious in high surface reactance. When including the reflected wave, the maximum excitation efficiency can be obtained approaches $95 \%$ when the surface reactance $X_{s}$ is around $j 250 \Omega$ as shown in Fig. 9 .

Finally, Fig. 10 shows the corresponding optimal normalized transducer aperture height $H_{a} / h_{e}$ as a function of surface reactance $X_{s}$. The $H_{a} / h_{e}$ ratio should be around 1.5 if the maximum TSW excitation efficiency, when $X_{s}$ is $j 250 \Omega$, is desirable. The ratio $H_{a} / \lambda_{0}$ is also included in Fig. 10 for easy estimation of the physical height. In this case, the $H_{a}$ is about $0.36 \lambda_{0}$, i.e. $1.8 \mathrm{~mm}$ at $60 \mathrm{GHz}$.

\section{Transverse field distribution at different frequency points}

As explained in [20], the transverse field distribution of a Zenneck wave does not vary with frequency. To demonstrate the wave described in this paper is indeed surface wave, we plot the transverse component of the E-field $(E z)$ at 30, 60 and 90 $\mathrm{GHz}$ along the propagation direction as shown in Fig. 11. When compared to Fig. 3 in [20], Fig. 11(a) shows a significant difference between the fields at the three frequency points. In addition, it can also be observed from Fig. 11(b) that the field becomes more concentrated to the dielectric/conductor interface when the frequency increases, which is agreed with the results in [20] as well. Therefore, the wave studied in this paper is surface wave, not Zenneck wave.

\section{Design of An Aperture Transducer with a full 3D- MODEL}

In Section II, the optimum surface reactance and transducer height combination for maximum TSW excitation efficiency have been discussed. When designing a practical transducer, the angular coverage of TSW in the horizontal plane has to be considered as well. Therefore in this section, a full 3D-model is built to study the relation between the angular coverage and the transducer width as shown in Fig. 12. A transducer with a

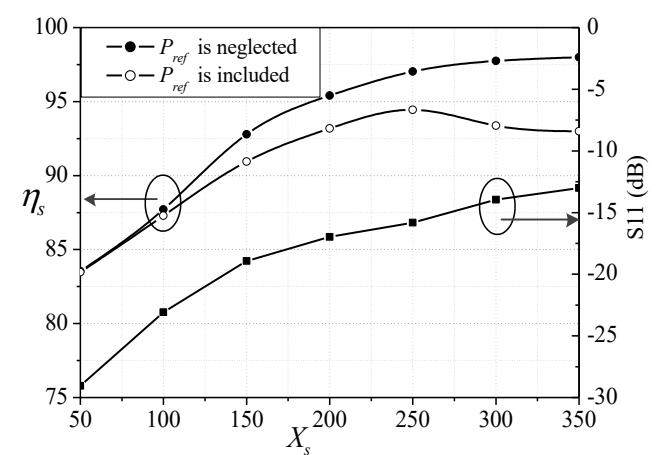

Fig. 9. TSW excitation efficiency as a function of surface reactance $X_{S}$.

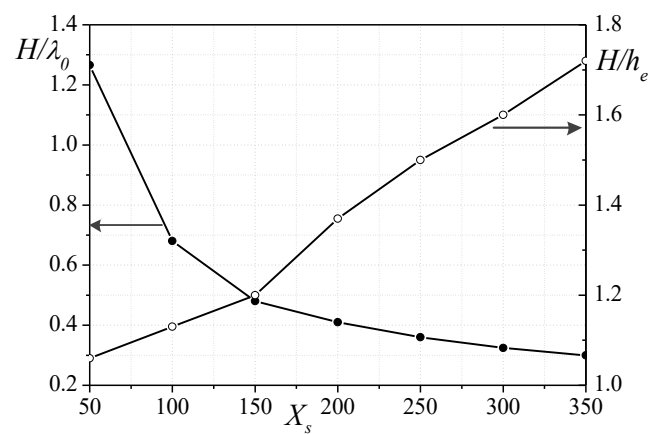

Fig. 10. The optimum aperture height as a function of surface reactance $X_{s}$. 


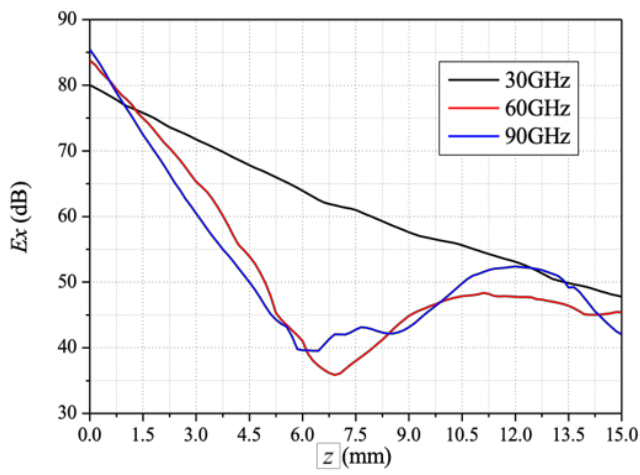

(a)

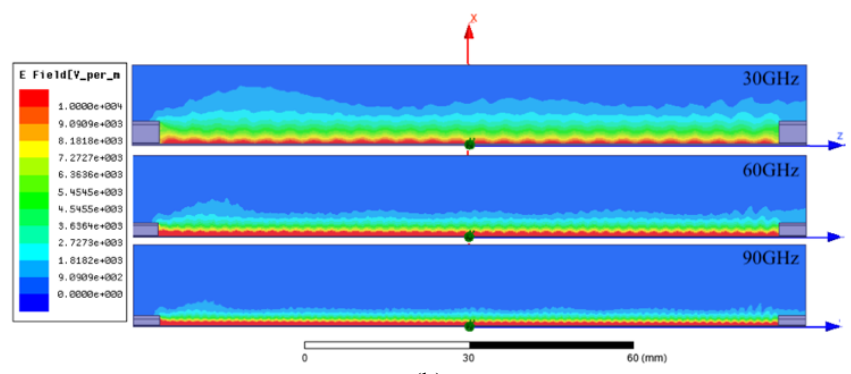

(b)

Fig. 11. Distribution of the transverse component of the reflected field (a) in the range from 0 to $15 \mathrm{~mm}$ (b) in $140 \mathrm{~mm}$ range.

rectangular aperture on a lossless reactive circular surface is considered. The radius of the circular surface is selected to be $20 \lambda_{0}$ to mimimize the reflection at the perimeter. The operating frequency is $60 \mathrm{GHz}\left(\lambda_{0}=5 \mathrm{~mm}\right)$ and surface reactance is chosen to be $j 250 \Omega$ which is the optimal value for maiximum excitation efficiency as illustrated in Fig. 9. Then we can evaluate $h_{e}=1.2 \mathrm{~mm}$ and $H_{a}=1.8 \mathrm{~mm}\left(0.36 \lambda_{0}\right)$ from (5) and Fig. 10 respectively.

\section{A. Effect of aperture width on the TSW excitation efficiency}

First, to further explain the validation of the computing TSW excitation efficiency of a pseudo 3D model, the effect of the aperture width $W_{a}$ on TSW excitation efficiency is studied in a full 3D EM simulation model. The calculated TSW excitation efficiency of a rectangular aperture transducer with three different aperture widths $\left(W_{a}=1.0 \lambda_{0}, 1.5 \lambda_{0}\right.$ and 2.0 $\left.\lambda_{0}\right)$ are listed in Table II. It is clear that the TSW excitation efficiency from the full 3D-model is close to that of the pesudo 3D one, i.e., 94.44\%. It also shows that the aperture width has less impact on the TSW excitation efficiency.

On the other hand, it is seen that the TSW excitation efficiency from the full 3D-model is sightly lower than that of the pesudo $3 \mathrm{D}$ one. This can be explained that in the pesudo model some space wave is reflected at the perfect magnetic boundaries, and the reflected space wave with incident angle larger than the Brewster angle to the reactive surface in the $x z$ planes will turn into TSW. However, this phenomenon does not occur in the 3D-model because free radiation boundaries are considered, thus the TSW excitation efficiency from the 3Dmodel will slightly be less than that from the pesudo model. Moreover, the $E$-field distribution with three different aperture width of the transducer $W_{a}$ in the $y z$-plane at $x=0$ are illustrated in Fig. 12. It can be observed that an aperture transducer produces a directional coverage, and the coverage angle $\phi_{0}$ is basically inversely proportional to the transducer width. Therefore, less energy propagates toward the $x z$-planes in

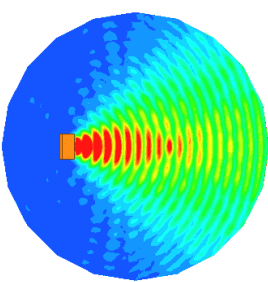

(a)

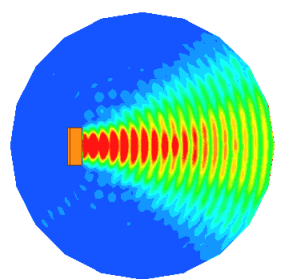

(b)

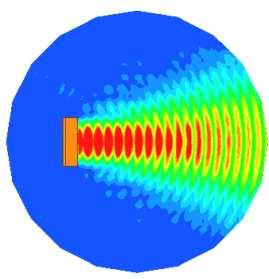

(c)
Fig. 12. Magnitude of $E$-field in the horizontal plane (a) $W_{a}=1.0 \lambda_{0}$ (b) $W_{a}=1.5 \lambda_{0}$ (c) $W_{a}=2.0 \lambda_{0}$ (the color scale is shown in Fig. 15).

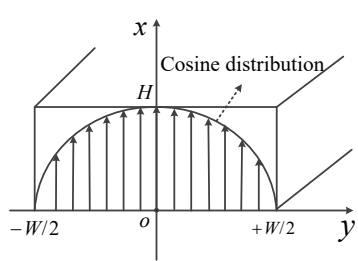

(a)

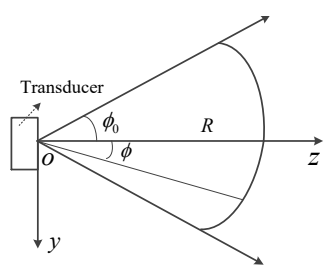

(b)
Fig. 13. A rectangular aperture transducer and its angular coverage (a) A rectangular aperture transducer with cosine $E$-field distribution in $x y$-plane, (b) The TSW angular coverage of a rectangular aperture transducer (top view).

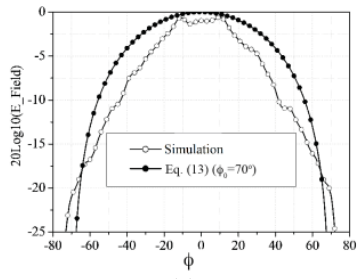

(a)

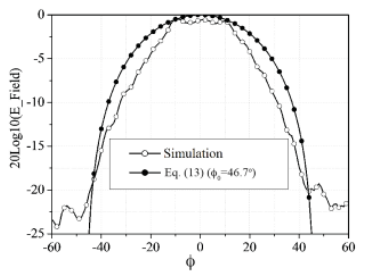

(b)

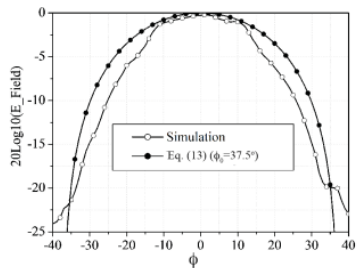

(c)

Fig. 14. E-field distribution in the $y z$-plane at $x=0$ for different aperture width, (a) $W_{a}=1.0 \lambda_{0}$, (b) $W_{a}=1.5 \lambda_{0}$, (c) $W_{a}=2.0 \lambda_{0}$

pesudo 3D-model, and the TSW excitation efficiency gradually approaches to the results of pesudo 3D-model as the aperture width increases.

TABLE II TSW EXCITATION EFFICIENCY AGAINST THE WIDTH OF APERTURE TRANSDUCER

\begin{tabular}{|c|c|c|c|}
\hline No. & $\begin{array}{c}\text { Aperture } \\
\text { Width }\left(W_{a}\right)\end{array}$ & in term of $\lambda_{0}$ & Efficiency \\
\hline 1 & $5.0 \mathrm{~mm}$ & 1.0 & $87.83 \%$ \\
\hline 2 & $7.5 \mathrm{~mm}$ & 1.5 & $92.25 \%$ \\
\hline 3 & $10.0 \mathrm{~mm}$ & 2.0 & $93.12 \%$ \\
\hline
\end{tabular}

\section{B. TSW angular coverage in the horizontal plane}

When the transducer is excited by fundamental $\mathrm{TE}_{10}$ mode with cosine $E$-field distribution over the transducer width as shown in Fig. 13(a), the excited TSW $E$-fields remain the cosine variation in the $\phi$-direction over the reactive surface. To establish the relationship between the angular coverage and aperture width, a cosine function is highly suitable to fit the $E$ field distribution in the $\phi$-direction. Therefore, the $E$-field propagation outside the rectangular transducer aperture can be approximately expressed as the following relationship: 


$$
E_{\phi}=E_{\phi_{0}} \cos \frac{\phi \pi}{2 \phi_{0}}
$$

where $E_{\phi_{0}}$ is the amplitude of E-field in the $\phi$-direction, $\phi_{0}$ is the half angle of the TSW beam and $\phi_{0} \leq \pi$. In (12), it is assumed that the TSW $E$-field is only inside the range of $\left[-\phi_{0,+}\right.$ $\left.\phi_{0}\right]$ and is null outside $\phi_{0}$, as shown in Fig. 13. $\phi_{0}$ is related to the aperture width. To extract the value of $\phi_{0}$, three sample aperture widths, $W_{a}=1.0 \lambda_{0}, 1.5 \lambda_{0}$ and $2.0 \lambda_{0}$ are applied, as shown in Fig. 14. Then we can obtain

$$
\phi_{0}=70 \frac{\lambda}{W_{a}}\left(^{\circ}\right)
$$

From Fig. 14, it can be observed that (13) agrees reasonably well with the $E$-field distribution above $-15 \mathrm{~dB}$. Further, using (14), we can obtain the half-power angular coverage $\phi_{3 \mathrm{~dB}}$

$$
\phi_{3 \mathrm{~dB}}=\phi_{0}=70 \frac{\lambda}{W_{a}}\left(^{\circ}\right)
$$

\section{Effect of aperture height on the TSW excitation efficiency}

The width of the transducer aperture, $W_{a}$, is fixed at the value of $7.5 \mathrm{~mm}$, i.e., $1.5 \lambda_{0}$ at $60 \mathrm{GHz}$, and the apeture height of the transducer $H_{a}$ is changed. The $E$-field distribution with three different $H_{a}\left(0.08 \lambda_{0}, 0.36 \lambda_{0}\right.$ and $\left.0.56 \lambda_{0}\right)$ in the $x z$-plane are shown in Fig. 15. Fig. 16 shows the TSW excitation efficiency of different rectangular transducer with the height varying from $0.08 \lambda_{0}$ to $0.56 \lambda_{0}$. The maximum TSW excitation efficiency, $92 \%$, occurs when the height is $0.36 \lambda_{0}\left(1.5 h_{e}\right)$ which is consistent with the results from the pseduo 3D model. If the transducer height displaces from the best value, the excitation efficiency will drop. It can be explained that when the surface reactance and operating frequency are given, the decay constant $\alpha_{1}$ can be determined by (2), and the $E$-field distribution on the reactive surface will be defined, the maximum TSW excitation efficiency will occur when the aperture $E$-field distrubution has

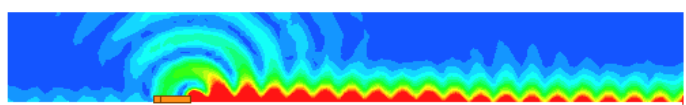

(a)

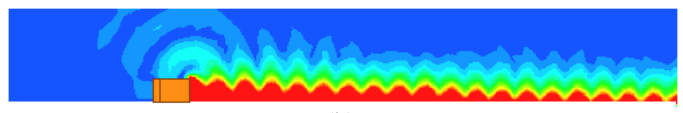

(b)

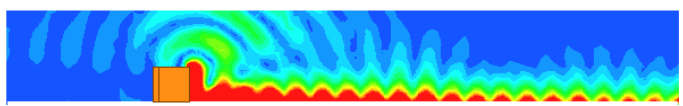

(c)

Fig. 15. The $E$-field distribution in the $x z$-plane of different aperture height, (a) $H_{a}=0.08 \lambda_{0}$, (b) $H_{a}=0.36 \lambda_{0}$, and (c) $H_{a}=0.56 \lambda_{0}$

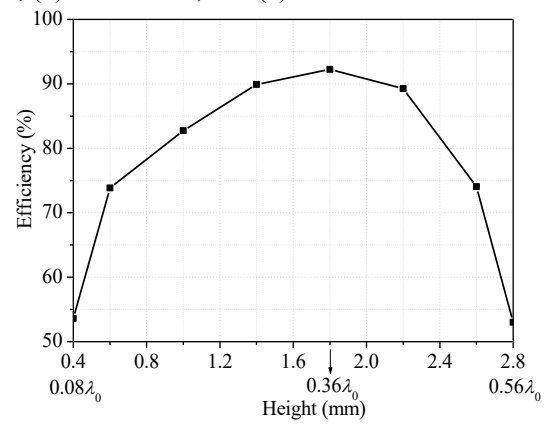

Fig. 16. The calcuated TSW excitation efficiency as a function of the transducer apeture height $H_{a}$ in the 3D model. the best matching status with the TSW $E$-field distrubution. The optimal tranducer height can be found in Fig. 10. If the transducer height displaces from the best value, more space wave will be incurred due to the severer mismatch between the $E$-field distribution in the rectangular transducer aperture and that propagating on the reactive surface and thus decreases the excitation efficiency.

Meanwhile, the transducer shows the TSW excitation efficiency better than $50 \%$, i.e. $3 \mathrm{~dB}$ when compared to input power $\left(P_{t o t}\right)$, from $0.08 \lambda_{0}$ to $0.60 \lambda_{0}$, which indicates its wide operating frequency band feature.

\section{EXPERIMENTAL VERIFICATION}

To verify the proposed theoretical study, in addition to the full 3D EM model including the two TSW transducers of the properly designed aperture and the corresponding TSW plane, an experiment setup as shown in Fig. 17 was built. The two TSW transducers with the aperture dimensions based on the theoretical results shown in Section II centered at $60 \mathrm{GHz}$ for covering the millimeter-wave frequency range are designed. The separation between the two transducers was fixed at 140 $\mathrm{mm}\left(28 \lambda_{0}\right.$ at $\left.60 \mathrm{GHz}\right)$ for the measurement. A Keysight N5250A-017 millimeter-wave network analyzer available at City University of Hong Kong was used for the transmission $\left(\mathrm{S}_{21}\right)$ test of the surface wave platform. The authors would like to point out that it is impossible to realize the virtual waveguide ports described in Section II. To achieve the highest accuracy, the network analyzer was first calibrated and normalized to eliminate the cable and TSW transducers effects, i.e., $\mathrm{S}_{21}$ equals $0 \mathrm{~dB}$ when the two transducers were directly connected as shown in Fig. 18. Then the two transducers are placed on a piece of conventional single-side microwave substrate. Taconic TLY -5 microwave substrate $\left(\varepsilon_{\mathrm{r}}=2.2\right.$, thickness $=0.38 \mathrm{~mm}, 300$ $\times 300 \mathrm{~mm}^{2}, \tan \delta=0.0009$ at $10 \mathrm{GHz}$,) was selected based on the surface impedance $(X s)$ it can provide and the low dielectric loss when compared to other microwave substrates available in hand. The surface impedance of the Taconic TLY-5 substrate across the millimeter-wave frequency band can be evaluated by (16) and (17) described in [9].

$$
X_{s}=2 \pi f \mu_{0}\left[\frac{\varepsilon_{r}-1}{\varepsilon_{r}} h_{s u b}+\frac{\Delta}{2}\right]
$$

where the skin depth of a copper sheet, $\Delta$, is

$$
\Delta=\sqrt{\frac{1}{\pi f \mu_{0} \sigma}}
$$

The dielectric substrate is of thickness, $h_{s u b}$, and dielectric constant, $\varepsilon r$. the operating frequency, $f$, and conductivity $\sigma$. Fig. 19 shows the variation of the surface impedance between 10 to $100 \mathrm{GHz}$. At $60 \mathrm{GHz}$, the surface impedance of the TSW platform equals $j 100 \Omega$. Although it is not the optimal $j 250 \Omega$ impedance, it demonstrates the guidelines for determining the initial transducer aperture size. In addition, the authors would like to mention that there are a few more practical constraints when designing the transducer, such as the coaxial-towaveguide excitation adaptor, the impedance bandwidth of the transducer and the mechanical support (e.g. the flange). In particular, there is an $0.1 \mathrm{~mm}$ gap $(\mathrm{g})$ between the bottom of the aperture and the top of the dielectric layer. Therefore, the transducer aperture was finely optimized in the 3D simulation model based on the theoretical results provided by the study. 
Fig. 20 shows the 3D EM simulation model of the transducer and the notations for main dimensions. The detailed dimensions of the transducers have been tabulated in Table III.

TABLE III. DIMENSIONS OF THE APERTURE TRANSDUCER (MILLIMETERS)

\begin{tabular}{|l|l|l|}
\hline Width of aperture & $\mathrm{W}_{\mathrm{a}}$ & $9.6\left(1.92 \lambda_{0}\right)$ \\
\hline Height of aperture & $\mathrm{H}_{\mathrm{a}}$ & $2.8\left(1.0 h_{e}\right)$ \\
\hline $\begin{array}{l}\text { Separation between the bottom of the aperture } \\
\text { to the top of the substrate }\end{array}$ & $\mathrm{g}$ & 0.1 \\
\hline Width of flange & $\mathrm{W}_{\mathrm{f}}$ & 28.1 \\
\hline Height of flange & $\mathrm{H}_{\mathrm{f}}$ & 13.4 \\
\hline
\end{tabular}

The measured transmission on the TSW platform is shown in Fig. 21. It can be observed that the $1 \mathrm{~dB}-$, and $3 \mathrm{~dB}-$ transmission-bandwidth is about $29.7 \mathrm{GHz}$ (from 37.9 to 67.6 $\mathrm{GHz}$ ) and $52 \mathrm{GHz}$ (from 34.4 to $86.4 \mathrm{GHz}$ ) respectively. Theoretically, such TSW platform can support communications systems requiring data rate in the order of tens of Gbps. Moreover, the average transmission over the $140 \mathrm{~mm}$ separation is about $-16 \mathrm{~dB}$, which is much higher than that of the free space communication. The transmission for the cases free space communication and over a piece of perfect electric conductor are also included for the readers' reference. The measured results generally follow the trend of the simulated results, both results match well with each other below $45 \mathrm{GHz}$, and the discrepancy starts to increase to about $1 \mathrm{~dB}$ up to 60 $\mathrm{GHz}$, but it increases further to $3 \mathrm{~dB}$ after $60 \mathrm{GHz}$. The authors suspect such difference may cause by the relative higher dielectric loss at higher frequency in the microwave substrate. Also, as explained, the surface impedance of the presented model is only $j 100 \Omega$, rather than the optimal $j 250 \Omega$, it is also expected that the transmission will be higher if the TSW platform is realized on a proper microwave substrate, although the transmission bandwidth will be of similar order.

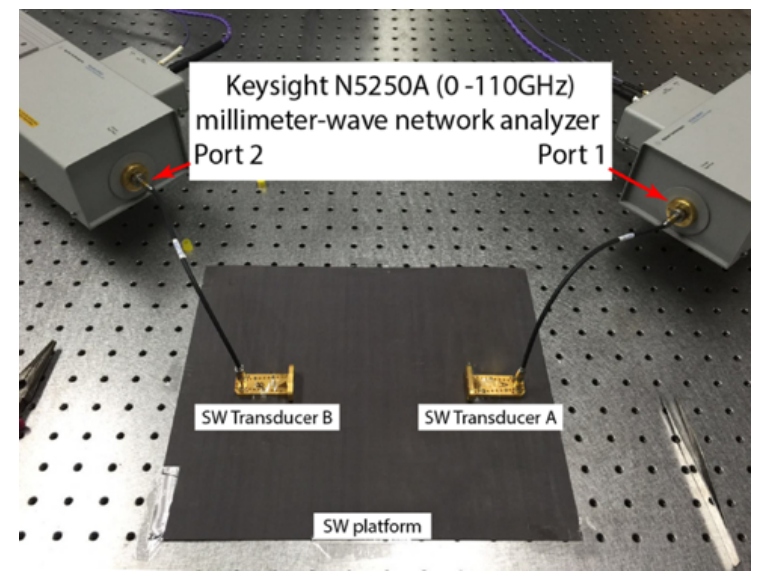

Fig. 17. The measurement setup of the propagation loss $\left(\mathrm{S}_{21}\right)$.

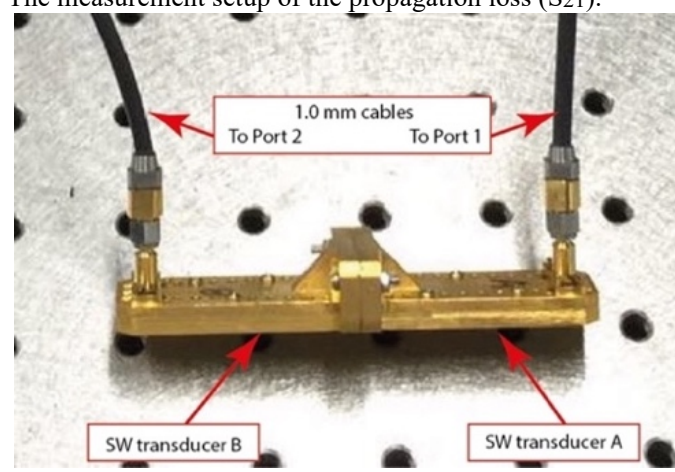

Fig. 18. The calibration setup for the TSW measurement.

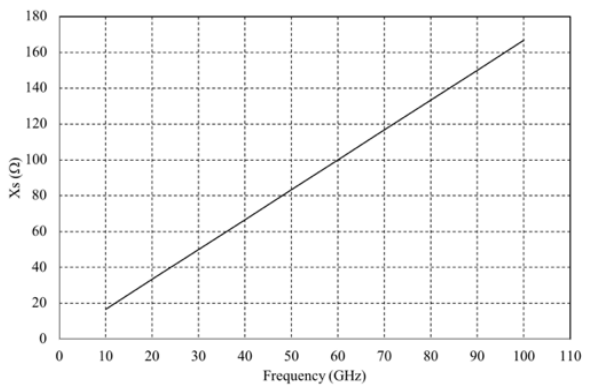

Fig. 19. The surface impedance of the Taconic TLY-5 microwave substrate.

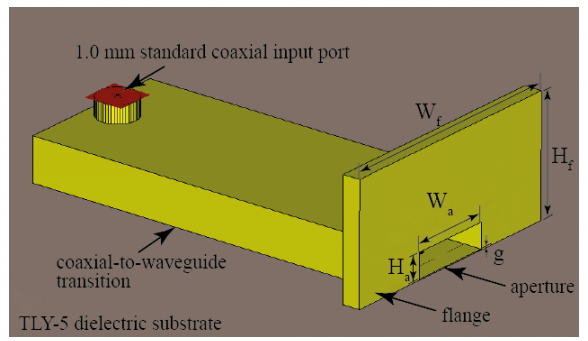

Fig. 20. The 3D EM simulation model of the TSW transducer.

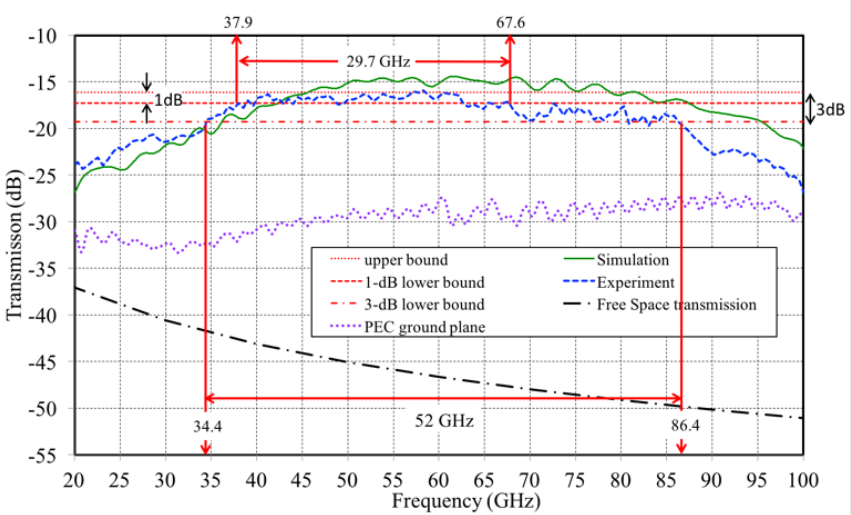

Fig. 21 Comparison of the simulated and measured $\mathrm{S}_{21}$.

\section{CONCLUSIONS}

This paper has introduced the design method of a rectangular aperture transducer on a wideband reactive surface, a validation method for computing the TSW excitation efficiency based on the TSW theory and 3D electromagnetic simulation software is derived. The method is applicable for arbitrary reactive surface of different impedance and transducers. Then the paper focuses on finding the optimum aperture height for maximum TSW excitation efficiency. The TSW excitation efficiencies of different reactive surfaces with various vertical aperture heights have been studied, and the maximum excitation efficiency and the corresponding optimum transducer aperture height $\left(H_{a}\right)$ are obtained. Further, a 3D-model is set up to determine the relationship between the angular coverage and the aperture width $\left(W_{a}\right)$. These studies show that the transducer aperture height causes impact on the TSW excitation efficiencies while the transducer aperture width affects the TSW angular coverage, the aperture parameters of a transducer can be determined independently. A $52 \mathrm{GHz}$ wide 3dB-transmission-bandwidth $\left(\mathrm{S}_{21}\right)$ was obtained. The simulation results from the 3D simulation model have been validated by the experimental results.

The surface wireless communications systems show many advantages, such as one-to-many (1-to-M) communication, 
power savings, excellent EMC characteristic, non-line-of-sight communication, and it is receiving more attention. This work will support the research on the TSW communications systems, including transducers, materials for reactive surfaces. It will speed up the applications of the TSW communications systems. Our future work will move onto the realization of practical wideband aperture transducers over low loss reactive surfaces. Besides, a test bed for wideband TSW communications systems will be also implemented to demonstrate the advantages of the TSW technology.

\section{ACKNOWLEDGEMENT}

The research visit of Dr. Wan at UCL was co-sponsored by the China Scholarship Council and Xi'an Institute of Space Radio Technology. This work was supported in part by the Hong Kong Research Grants Council (T42-103/16-N). We would also like to thank Dr. Kungbo $\mathrm{Ng}$ from the State Key Laboratory of Millimeter Waves at the City University of Hong Kong for his support in the experimental work.

\section{REFERENCES}

T. K. Sarkar, W. M. Dyab, M. N. Abdallah, M. Salazar-Palma, M. V. S. N. Prasad, and S.-W. Ting, 'Application of the Schelkunoff Formulation to the Sommerfeld Problem of a Vertical Electric Dipole Radiating Over an Imperfect Ground', IEEE Trans. Antennas Propag., vol. 62, no. 8, pp. 4162-4170, Aug. 2014.

[2] J. Zenneck, 'Propagation of plane EM waves along a plane conducting surface', Ann. Phys.(Leipzig), 1907.

[3] T. K. . Sarkar, W. Dyab, M. N. . Abdallah, M. Salazar-Palma, M. V. S. N. . Prasad, S. Barbin, and S. W. Ting, 'Physics of propagation in a cellular wireless communication environment', URSI Radio Sci. Bull., vol. 85, no. 4, p. 5, 2012.

[4] G. Rich, 'The launching of a plane surface wave', Proc. IEE - Part B Radio Electron. Eng., vol. 102, no. 2, pp. 237-246, Mar. 1955.

[5] W. M. G. Fernando and H. E. M. Barlow, 'An investigation of the properties of radial cylindrical surface waves launched over flat reactive surfaces', Proc. IEE - Part B Radio Electron. Eng., vol. 103, no. 9, pp. 307-318, May 1956.

[6] K. P. Sharma, 'An investigation of the excitation of radiation by surface waves', Proc. IEE - Part B Radio Electron. Eng., vol. 106, no. 26, pp. 116-122, Mar. 1959.

[7] K. P. Sharma, 'The estimation of the reactance of a loss-free surface supporting surface waves', Proc. IEE - Part B Electron. Commun. Eng., vol. 106, no. 28, pp. 427-430, Jul. 1959.

[8] A. L. Cullen and J. A. Staniforth, 'The launching of surface waves by an end-fire array of slots', Proc. IEE Part C Monogr., vol. 108, no. 14 , p. 492, 1961.

[9] H. M. Barlow and Brown John, Radio Surface Waves. Clarendon P, 1962.

[10] E. Johansen, 'Surface wave scattering by a step', IEEE Trans. Antennas Propag., vol. 15, no. 3, pp. 442-448, May 1967.

[11] D. Hemmendinger and F. Zucker, 'A forgotten theorem, and its application to surface-wave excitation', IEEE Trans. Antennas Propag., vol. 18, no. 1, pp. 132-133, Jan. 1970.

[12] D. A. Hill and J. R. Wait, 'Excitation of the Zenneck surface wave by a vertical aperture', Radio Sci., vol. 13, no. 6, pp. 969-977, 1978. G. L. James, 'Surface reactance of corrugated planes', Electron. Lett., vol. 15, no. 23, p. 751, 1979.

[14] J. LaComb and P. Mileski, 'Ultra Wideband Surface Wave Communication', Prog. Electromagn., vol. 8, pp. 95-105, 2009.

[15] J. E. Turner, M. S. Jessup, and K. F. Tong, 'A novel technique enabling the realisation of $60 \mathrm{GHz}$ body area networks', Proc. BSN 2012 9th Int. Work. Wearable Implant. Body Sens. Networks, no. 1, pp. 58-62, 2012.

[16] A. J. Karkar, J. E. Turner, K. Tong, R. Ai-Dujaily, T. Mak, A. Yakovlev, and F. Xia, 'Hybrid wire-surface wave interconnects for next-generation networks-on-chip', IET Comput. Digit. Tech., vol. 7, no. 6, 2013.

[17] J. Turner and M. Jessup, 'How Surface Waves Reduce ThroughLife Costs', Meas. Control, vol. 46, no. 6, pp. 180-184, Jul. 2013.
'Periodic structures to efficiently launch HF surface waves', Proc. 5th Eur. Conf. Antennas Propag. EUCAP, pp. 655-657, 2011.

[19] Ansoft Corporation, 'User's guide - High Frequency Structure Simulator', 2005

[20] T. K. Sarkar, M. N. Abdallah, M. Salazar-Palma, and W. M. Dyab, 'Surface Plasmons/Polaritons, Surface Waves, and Zenneck Waves: Clarification of the terms and a description of the concepts and their evolution.', IEEE Antennas Propag. Mag., vol. 59, no. 3, pp. 77-93, 2017.

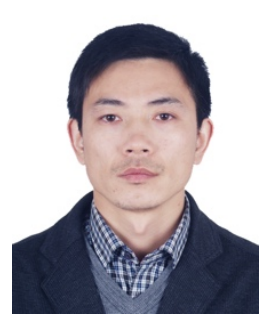

Jixiang Wan received the B.S., M.S. and Ph.D. degrees in Electronics Engineering from Xi'dian University, China, in 2000, 2003 and 2004 respectively. He joined Xi' an Institute of Space Radio Technology (XISRT), China, in 2005, where he was involved with the design of space antennas and over four satellite programs as a director of antenna subsystem. He is now a professor and associate director of Space Antenna Department in XISRT. During July 2014 and June 2015, he was a visiting scholar at the Department of Electronic and Electrical Engineering, UCL, where he was involved with millimeter-wave wireless communications systems based on surface wave technology. His research mostly involves $\mathrm{THz}$ antennas, multi-beam antennas, phased array antennas for space applications and RF front-end for surface wave wireless communications systems.

Dr. Wan has published over 30 papers in technical journals and conferences in the area of space antennas and satellite communications. He has 5 Chinese patents that have been awarded, and 2 Chinese patents that are pending. Dr. Wan was awarded the first prize for scientific and technological progress from China Aerospace Science and Technology (CAST) Group, 2017.

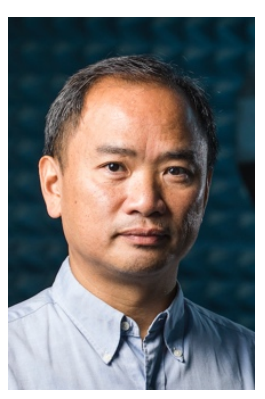

Kin Fai Tong (M'99-SM'13) received the B.Eng. and $\mathrm{Ph} . \mathrm{D}$. degrees in electronic engineering from the City University of Hong Kong in 1993 and 1997, respectively.

After graduation, Dr. Tong worked in the Department of Electronic Engineering at City University of Hong Kong as a Research Fellow. Two years later, he took up the post Expert researcher in the Photonic Information Technology Group and Millimetre-wave Devices Group at the National Institute of Information and Communications Technology (NiCT), Japan, where his main research focused on photonicmillimeter-wave planar antennas for high-speed wireless communications systems. In 2005, he started his academic career in the Department of Electronic and Electrical Engineering, UCL, as a lecturer. Now Dr. Tong is a Reader in Antennas, Microwave and Millimeter-wave Engineering in the department. His current research interests include millimeter-wave antennas, fluid antennas, 3D printed antennas and sub-GHz long range IoT networks. He served as the General Co-Chair of the 2017 International Workshop on Electromagnetics (iWEM).

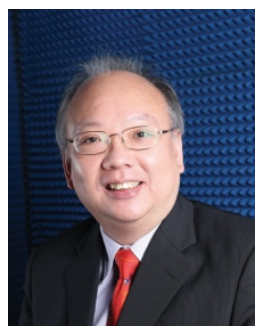

Chi Hou Chan (S'86-M'86-SM'00-F'02) received the B.S. and M.S. degrees in electrical engineering from the Ohio State University, Columbus, OH, USA, in 1981 and 1982, respectively, and the Ph.D. degree in electrical engineering from the University of Illinois, Urbana, IL, USA, in 1987.

From 1987 to 1989, Dr. Chan was a Visiting Assistant Professor in the Department of Electrical and Computer Engineering at the University of Illinois. From 1989 to 1998 , he was a faculty member in the Department of Electrical Engineering at the University of Washington, Seattle, WA, USA. In 1996, he joined the Department of Electronic Engineering, City University of Hong Kong, and was promoted to Chair Professor of Electronic Engineering in 1998. From 1998 to 2009, he was first Associate Dean and then Dean of College of Science and Engineering. He also served as Acting Provost of the university from July 2009 to September 2010. He is currently the Director of State Key Laboratory of Terahertz and Millimeter Waves (City University of Hong Kong). His current research interests include computational electromagnetics, millimeter-wave circuits and antennas, and terahertz science and technology. He served as the General CoChair of the 2010 International Symposium of Antennas and Propagation (ISAP), the 2011 International Workshop on Antenna Technology (iWAT), the 2013 International Workshop on Electromagnetics (iWEM), the 2015 IEEE International Conference on Computational Electromagnetics (ICCEM), ICCEM 2016, ICCEM 2018, and the 2017 Global Symposium on MillimeterWaves (GSMM). 